\title{
Existence of Nontrivial Solutions for Generalized Quasilinear Schrödinger Equations with Critical Growth
}

\author{
Quanqing $L i \mathbb{D},{ }^{1}$ Kaimin Teng $\mathbb{D},{ }^{2}$ and Xian $\mathrm{Wu}^{3}$ \\ ${ }^{1}$ Department of Mathematics, Honghe University, Mengzi, Yunnan 661100, China \\ ${ }^{2}$ Department of Mathematics, Taiyuan University of Technology, Taiyuan, Shanxi 030024, China \\ ${ }^{3}$ Department of Mathematics, Yunnan Normal University, Kunming, Yunnan 650092, China
}

Correspondence should be addressed to Kaimin Teng; tengkaimin2013@163.com

Received 12 September 2017; Revised 12 November 2017; Accepted 16 November 2017; Published 3 January 2018

Academic Editor: Ciprian G. Gal

Copyright (C) 2018 Quanqing Li et al. This is an open access article distributed under the Creative Commons Attribution License, which permits unrestricted use, distribution, and reproduction in any medium, provided the original work is properly cited.

We study the following generalized quasilinear Schrödinger equations with critical growth $-\operatorname{div}\left(g^{2}(u) \nabla u\right)+g(u) g^{\prime}(u)|\nabla u|^{2}+$ $V(x) u=\lambda f(x, u)+g(u)|G(u)|^{2^{*}-2} G(u), x \in \mathbb{R}^{N}$, where $\lambda>0, N \geq 3, g(s): \mathbb{R} \rightarrow \mathbb{R}^{+}$is a $C^{1}$ even function, $g(0)=1$, and $g^{\prime}(s) \geq 0$ for all $s \geq 0$, where $G(u):=\int_{0}^{u} g(t) d t$. Under some suitable conditions, we prove that the equation has a nontrivial solution by variational method.

\section{Introduction and Preliminaries}

Consider the following generalized quasilinear Schrödinger equations with critical growth:

$$
\begin{aligned}
& -\operatorname{div}\left(g^{2}(u) \nabla u\right)+g(u) g^{\prime}(u)|\nabla u|^{2}+V(x) u \\
& =\lambda f(x, u)+g(u)|G(u)|^{2^{*}-2} G(u), \quad x \in \mathbb{R}^{N},
\end{aligned}
$$

where $\lambda>0, N \geq 3, g(s): \mathbb{R} \rightarrow \mathbb{R}^{+}$is a $C^{1}$ even function, $g(0)=1$, and $g^{\prime}(s) \geq 0$ for all $s \geq 0$.

The equations are related to the existence of solitary wave solutions for quasilinear Schrödinger equations

$$
\begin{aligned}
i z_{t}= & -\Delta z+W(x) z-k(x,|z|) z \\
& -\Delta l\left(|z|^{2}\right) l^{\prime}\left(|z|^{2}\right) z,
\end{aligned}
$$

where $z: \mathbb{R} \times \mathbb{R}^{N} \rightarrow \mathbb{C}, W: \mathbb{R}^{N} \rightarrow \mathbb{R}$ is a given potential, $l: \mathbb{R} \rightarrow \mathbb{R}$, and $k: \mathbb{R}^{N} \times \mathbb{R} \rightarrow \mathbb{R}$ are suitable functions. The form of (2) has been derived as models of several physical phenomena corresponding to various types of $l(s)$. For instance, the case $l(s)=s$ models the time evolution of the condensate wave function in superfluid film $[1,2]$ and is called the superfluid film equation in fluid mechanics by Kurihara [1]. In the case $l(s)=(1+s)^{1 / 2}$, problem (2) models the self-channeling of a high-power ultra short laser in matter, the propagation of a high-irradiance laser in a plasma creates an optical index depending nonlinearly on the light intensity, and this leads to interesting new nonlinear wave equations; see [3-6]. For more physical motivations and more references dealing with applications, we can refer to [7$14]$ and references therein.

Set $z(t, x)=\exp (-i E t) u(x)$, where $E \in \mathbb{R}$ and $u$ is a real function. Then (2) can be reduced to the corresponding equation of elliptic type (see [15]):

$$
-\Delta u+V(x) u-\Delta l\left(u^{2}\right) l^{\prime}\left(u^{2}\right) u=f(x, u),
$$

$$
x \in \mathbb{R}^{N},
$$

where $f(x, u)=k(x,|u|) u$. If we take

$$
g^{2}(u)=1+\frac{\left[\left(l\left(u^{2}\right)\right)^{\prime}\right]^{2}}{2},
$$

then (1) turns into (3) (see [16]).

Moreover, problem (3) also arises in biological models and propagation of laser beams when $g(u)$ is a positive constant (see $[17,18])$. In (3), if we set $l(u)=u$, that is, 
$g^{2}(u)=1+2 u^{2}$, then we get the superfluid film equation in plasma physics:

$$
-\Delta u+V(x) u-\Delta\left(u^{2}\right) u=f(x, u), \quad x \in \mathbb{R}^{N} ;
$$

if we set $l(u)=(1+u)^{1 / 2}$, that is, $g^{2}(u)=1+u^{2} / 2\left(1+u^{2}\right)$, then we get the equation

$$
\begin{aligned}
- & \Delta u+V(x) u-\left[\Delta\left(1+u^{2}\right)^{1 / 2}\right] \frac{u}{2\left(1+u^{2}\right)^{1 / 2}} \\
= & f(x, u), \quad x \in \mathbb{R}^{N},
\end{aligned}
$$

which models the self-channeling of a high-power ultrashort laser in matter.

In the past, the research on the existence of solitary wave solutions of Schrödinger equations (2) is for some given special function $l(s)$. In this paper, we will use a unified new variable replacement to study (2), constructed by Shen and Wang in [16]. Define the energy functional associated with (1) by

$$
\begin{aligned}
I_{\lambda}(u)= & \frac{1}{2} \int_{\mathbb{R}^{N}} g^{2}(u)|\nabla u|^{2} d x+\frac{1}{2} \int_{\mathbb{R}^{N}} V(x) u^{2} d x \\
& -\lambda \int_{\mathbb{R}^{N}} F(x, u) d x-\frac{1}{2^{*}} \int_{\mathbb{R}^{N}}|G(u)|^{2^{*}} d x,
\end{aligned}
$$

where $F(x, u):=\int_{0}^{u} f(x, t) d t$. However, $I_{\lambda}$ is not well defined in $H^{1}\left(\mathbb{R}^{N}\right)$ because of the term $\int_{\mathbb{R}^{N}} g^{2}(u)|\nabla u|^{2} d x$. To overcome this difficulty, we make a change of variable constructed by Shen and Wang in [16]: $v:=G(u):=\int_{0}^{u} g(t) d t$. Then we obtain

$$
\begin{aligned}
J_{\lambda}(v)= & \frac{1}{2} \int_{\mathbb{R}^{N}}|\nabla v|^{2} d x+\frac{1}{2} \int_{\mathbb{R}^{N}} V(x) G^{-1}(v)^{2} d x \\
& -\lambda \int_{\mathbb{R}^{N}} F\left(x, G^{-1}(v)\right) d x-\frac{1}{2^{*}} \int_{\mathbb{R}^{N}}|v|^{2^{*}} d x .
\end{aligned}
$$

If $u$ is a nontrivial solution of (1), then

$$
\begin{aligned}
& \left\langle I_{\lambda}^{\prime}(u), \varphi\right\rangle=\int_{\mathbb{R}^{N}}\left[g^{2}(u) \nabla u \nabla \varphi+g(u) g^{\prime}(u)|\nabla u|^{2} \varphi\right. \\
& \quad+V(x) u \varphi-\lambda f(x, u) \varphi \\
& \left.\quad-g(u)|G(u)|^{2^{*}-2} G(u) \varphi\right] d x=0
\end{aligned}
$$

for all $\varphi \in C_{0}^{\infty}\left(\mathbb{R}^{N}\right)$. Let $\varphi=(1 / g(u)) \psi$. By [16] we know that (9) is equivalent to

$$
\begin{gathered}
\left\langle J_{\lambda}^{\prime}(v), \psi\right\rangle=\int_{\mathbb{R}^{N}}\left[\nabla v \nabla \psi+V(x) \frac{G^{-1}(v)}{g\left(G^{-1}(v)\right)} \psi\right. \\
\left.-\lambda \frac{f\left(x, G^{-1}(v)\right)}{g\left(G^{-1}(v)\right)} \psi-|v|^{*^{*}-2} v \psi\right] d x=0
\end{gathered}
$$

for all $\psi \in C_{0}^{\infty}\left(\mathbb{R}^{N}\right)$. Therefore, in order to find the nontrivial solution of (1), it suffices to study the existence of the nontrivial solutions of the following equations:

$$
\begin{aligned}
- & \Delta v+V(x) \frac{G^{-1}(v)}{g\left(G^{-1}(v)\right)}-\lambda \frac{f\left(x, G^{-1}(v)\right)}{g\left(G^{-1}(v)\right)}-|v|^{2^{*}-2} v \\
& =0 .
\end{aligned}
$$

Recently, the authors studied generalized quasilinear Schrödinger equations with subcritical growth $[19,20]$, critical growth [21], and supercritical growth [22].

In order to reduce the statements for main results, we list the assumptions as follows:

$\left(V_{1}\right) V(x) \geq V_{0}:=\inf _{x \in \mathbb{R}^{N}} V(x)>0$ for all $x \in \mathbb{R}^{N}$.

$\left(V_{2}\right) \lim _{|x| \rightarrow \infty} V(x)=V_{\infty}<+\infty$ and $V(x) \leq V_{\infty}$ for all $x \in \mathbb{R}^{N}$.

$\left(f_{1}\right) f \in C\left(\mathbb{R}^{N} \times \mathbb{R}, \mathbb{R}\right)$ and there exists $2<p<2^{*}$ such that

$$
|f(x, t)| \leq C\left(1+g(t)|G(t)|^{p-1}\right)
$$

for all $(x, t) \in \mathbb{R}^{N} \times \mathbb{R}$.

$\left(f_{2}\right) f(x, t)=o(|t|)$ uniformly in $x \in \mathbb{R}^{N}$ as $|t| \rightarrow 0$.

$\left(f_{3}\right)\left(f\left(x, G^{-1}(t)\right) / g\left(G^{-1}(t)\right)\right) t \quad-2 F\left(x, G^{-1}(t)\right) \quad \geq$ $\left(f\left(x, G^{-1}(s t)\right) / g\left(G^{-1}(s t)\right)\right) s t-2 F\left(x, G^{-1}(s t)\right)$ for all $t \in \mathbb{R}$ and $s \in[0,1]$.

$\left(f_{4}\right) f(x, t) t>0$ for all $(x, t) \in \mathbb{R}^{N} \times \mathbb{R} \backslash\{0\}$.

$\left(f_{5}\right) \lim _{|t| \rightarrow+\infty}\left(F\left(x, G^{-1}(t)\right) / t^{2}\right)=+\infty$ uniformly in $x \in$ $\mathbb{R}^{N}$.

Set $E=H^{1}\left(\mathbb{R}^{N}\right)$ with the norm

$$
\|u\|_{E}=\left[\int_{\mathbb{R}^{N}}\left(|\nabla u|^{2}+u^{2}\right) d x\right]^{1 / 2} .
$$

It is easy to prove that $J_{\lambda}$ is well defined on $E$ and $J_{\lambda} \in$ $C^{1}(E, \mathbb{R})$ under our assumptions and its Gateaux derivative is given by

$$
\begin{gathered}
\left\langle J_{\lambda}^{\prime}(v), \varphi\right\rangle=\int_{\mathbb{R}^{N}}\left[\nabla v \nabla \varphi+V(x) \frac{G^{-1}(v)}{g\left(G^{-1}(v)\right)} \varphi\right. \\
\left.-\lambda \frac{f\left(x, G^{-1}(v)\right)}{g\left(G^{-1}(v)\right)} \varphi-|v|^{2^{*}-2} v \varphi\right] d x
\end{gathered}
$$

for all $v, \varphi \in E$.

Our main result of this paper is as follows.

Theorem 1. Suppose that $\left(V_{1}\right),\left(V_{2}\right)$, and $\left(f_{1}\right)-\left(f_{5}\right)$ are satisfied. Then if $N \geq 5$, (1) admits a nontrivial solution for all $\lambda>0$; if $N=3,4$, (1) admits a nontrivial solution for large $\lambda$.

Remark 2. Condition $\left(f_{3}\right)$ is weaker than the following condition $\left(f_{6}\right)$. 
$\left(f_{6}\right) f\left(x, G^{-1}(t)\right) / g\left(G^{-1}(t)\right) t$ is nonincreasing on $(-\infty, 0)$ and nondecreasing on $(0,+\infty)$.

Indeed, set $l(s)=s^{2} t\left(f\left(x, G^{-1}(t)\right) / g\left(G^{-1}(t)\right)\right)-$ $2 F\left(x, G^{-1}(s t)\right), \forall s \in[0,1]$. Then

$$
\begin{aligned}
l^{\prime}(s) & =2 s t \frac{f\left(x, G^{-1}(t)\right)}{g\left(G^{-1}(t)\right)}-2 \frac{f\left(x, G^{-1}(s t)\right)}{g\left(G^{-1}(s t)\right)} t \\
& =2 s t \frac{f\left(x, G^{-1}(t)\right)}{g\left(G^{-1}(t)\right)}-2 t \frac{f\left(x, G^{-1}(s t)\right)}{g\left(G^{-1}(s t)\right) s t}(s t) .
\end{aligned}
$$

If $\left(f_{6}\right)$ holds, then

$$
l^{\prime}(s) \geq 2 s t \frac{f\left(x, G^{-1}(t)\right)}{g\left(G^{-1}(t)\right)}-2 t \frac{f\left(x, G^{-1}(t)\right)}{g\left(G^{-1}(t)\right) t}(s t)=0
$$

whenever $t>0$ or $t<0$. Hence $l(s)$ is nondecreasing on $[0,1]$, and hence $l(1) \geq l(s)$ for all $s \in[0,1]$. Consequently, $\left(f_{6}\right)$ implies that

$$
\begin{aligned}
& t \frac{f\left(x, G^{-1}(t)\right)}{g\left(G^{-1}(t)\right)}-2 F\left(x, G^{-1}(t)\right) \\
& \quad \geq s^{2} t \frac{f\left(x, G^{-1}(t)\right)}{g\left(G^{-1}(t)\right)}-2 F\left(x, G^{-1}(s t)\right) \\
& \quad=s^{2} t|t| \frac{f\left(x, G^{-1}(t)\right)}{g\left(G^{-1}(t)\right)|t|}-2 F\left(x, G^{-1}(s t)\right) \\
& \quad \geq s^{2} t|t| \frac{f\left(x, G^{-1}(s t)\right)}{g\left(G^{-1}(s t)\right)|s t|}-2 F\left(x, G^{-1}(s t)\right) \\
& \quad=s t \frac{f\left(x, G^{-1}(s t)\right)}{g\left(G^{-1}(s t)\right)}-2 F\left(x, G^{-1}(s t)\right)
\end{aligned}
$$

for all $s \in[0,1]$; that is, the condition $\left(f_{3}\right)$ holds.

From Remark 2 we obtain Corollary 3.

Corollary 3. Suppose that $\left(V_{1}\right),\left(V_{2}\right),\left(f_{1}\right)-\left(f_{2}\right),\left(f_{4}\right)-\left(f_{5}\right)$, and $\left(f_{6}\right)$ are satisfied. Then if $N \geq 5$, (1) admits a nontrivial solution for all $\lambda>0$; if $N=3,4$, (1) admits a nontrivial solution for large $\lambda$.

Remark 4. In [16], Shen and Wang studied the existence of nontrivial solutions for generalized quasilinear Schrödinger equations

$$
\begin{aligned}
& -\operatorname{div}\left(g^{2}(u) \nabla u\right)+g(u) g^{\prime}(u)|\nabla u|^{2}+V(x) u \\
& =h(u), \quad x \in \mathbb{R}^{N},
\end{aligned}
$$

where $h$ is a subcritical nonlinearity satisfying the following conditions:

$$
\begin{aligned}
& \left(h_{0}\right) h(t)=0 \text { if } t \leq 0 . \\
& \left(h_{1}\right) h(t)=o(t) \text { as } t \rightarrow 0^{+} .
\end{aligned}
$$

$\left(h_{2}\right)$ There exists $2<p<2^{*}$ such that

$$
|h(t)| \leq C\left(1+g(t)|G(t)|^{p-1}\right)
$$

for all $t>0$.

$\left(h_{3}\right)$ There exists $\mu>2$ such that, for any $t>0$, there holds

$$
0<\mu g\left(G^{-1}(t)\right) H\left(G^{-1}(t)\right) \leq h\left(G^{-1}(t)\right) t .
$$

As mentioned above, if we set $g^{2}(u)=1+2 u^{2}$, then we get the superfluid film equation in plasma physics

$$
-\Delta u+V(x) u-\Delta\left(u^{2}\right) u=h(u), \quad x \in \mathbb{R}^{N},
$$

whose nontrivial solutions were studied in [23]. But our problem (1) is elliptic problem involving the critical exponent, so our result extends the results of the work $[16,23]$ to a critical setting. Moreover, the assumptions about the nonlinearity in this paper are different from the assumptions about the nonlinearity in $[16,23]$.

Remark 5. In [24], Deng et al. studied problem (1) and their result based on more harsh conditions:

$\left(f_{1}\right)^{*} f(x, t) \geq 0$ is differentiable with respect to $t \in$ $[0,+\infty)$ for all $x \in \mathbb{R}^{N}$ and continuous with respect to $x \in \mathbb{R}^{N}$ for all $t \in[0,+\infty)$. Moreover, $f(x, t) \equiv 0$ for all $(x, t) \in \mathbb{R}^{N} \times \mathbb{R}^{-}$.

$\left(f_{3}\right)^{*}$ There exists $\delta \in\left(0,2^{*}-2\right)$ such that, for any $t>$ 0 , there holds $(1+\delta) f(x, t) \leq G(t)[f(x, t) / g(t)]^{\prime}$, which implies that there exists $\mu \in\left(2,2^{*}\right)$ such that $f(x, t) G(t) \geq \mu g(t) F(x, t)$ for all $(x, t) \in \mathbb{R}^{N} \times \mathbb{R}$.

In this paper, we just assume that $f$ is a continuous function. Moreover, there are functionals $f(x, t)$ satisfying $\left(f_{3}\right)$ but not satisfying the above Ambrosetti-Rabinowitz type condition (see Remark 1.2 in [25]). Hence, our result is different from the result there.

\section{Proof of Theorem 1}

To begin with, we give some lemmas.

Lemma 6. For the functions $g, G$, and $G^{-1}$, the following properties hold:

(1) the functions $G(\cdot)$ and $G^{-1}(\cdot)$ are strictly increasing and odd;

(2) $G(s) \leq g(s) s$ for all $s \geq 0 ; G(s) \geq g(s) s$ for all $s \leq 0$;

(3) $g\left(G^{-1}(s)\right) \geq g(0)=1$ for all $s \in \mathbb{R}$;

(4) $G^{-1}(s) / s$ is decreasing on $(0,+\infty)$ and increasing on $(-\infty, 0)$;

(5) $\left|G^{-1}(s)\right| \leq(1 / g(0))|s|=|s|$ for all $s \in \mathbb{R}$;

(6) $\left|G^{-1}(s)\right| / g\left(G^{-1}(s)\right) \leq\left(1 / g^{2}(0)\right)|s|=|s|$ for all $s \in \mathbb{R}$;

(7) $G^{-1}(s) s / g\left(G^{-1}(s)\right) \leq\left|G^{-1}(s)\right|^{2}$ for all $s \in \mathbb{R}$; 
(8) $\lim _{|s| \rightarrow 0}\left(G^{-1}(s) / s\right)=1 / g(0)=1$ and

$$
\lim _{|s| \rightarrow \infty} \frac{G^{-1}(s)}{s}= \begin{cases}\frac{1}{g(\infty)}, & \text { if } g \text { is bounded, } \\ 0, & \text { if } g \text { is unbounded. }\end{cases}
$$

Proof. Properties (1)-(3) are obvious. By (2), we have

$$
\left(\frac{G^{-1}(s)}{s}\right)^{\prime}=\frac{s-G^{-1}(s) g\left(G^{-1}(s)\right)}{g\left(G^{-1}(s)\right) s^{2}} \leq 0
$$

for all $s>0$ and

$$
\left(\frac{G^{-1}(s)}{s}\right)^{\prime}=\frac{s-G^{-1}(s) g\left(G^{-1}(s)\right)}{g\left(G^{-1}(s)\right) s^{2}} \geq 0
$$

for all $s<0$. Consequently, we obtain (4). By mean value theorem and (3), one has

$$
\begin{aligned}
\left|G^{-1}(s)\right| & =\left|G^{-1}(s)-G^{-1}(0)\right|=\frac{1}{g\left(G^{-1}(\theta s)\right)}|s| \\
& \leq \frac{1}{g(0)}|s|
\end{aligned}
$$

for all $s \in \mathbb{R}$, where $\theta \in(0,1)$; that is, (5) is proved. Obviously, (6) is a consequence of (3) and (5). Moreover, (7) is a consequence of (2). Finally, using L' Hospital's rule, we know that (8) is satisfied. This completes the proof.

Denote

$$
\begin{aligned}
h_{\lambda}(x, s)= & V(x) s-V(x) \frac{G^{-1}(s)}{g\left(G^{-1}(s)\right)} \\
& +\lambda \frac{f\left(x, G^{-1}(s)\right)}{g\left(G^{-1}(s)\right)} .
\end{aligned}
$$

Then

$$
\begin{aligned}
H_{\lambda}(x, s) & :=\int_{0}^{s} h_{\lambda}(x, t) d t \\
& =\frac{1}{2} V(x)\left[s^{2}-G^{-1}(s)^{2}\right]+\lambda F\left(x, G^{-1}(s)\right) .
\end{aligned}
$$

Consequently,

$$
\begin{aligned}
J_{\lambda}(v)= & \frac{1}{2} \int_{\mathbb{R}^{N}}\left[|\nabla v|^{2}+V(x) v^{2}\right] d x \\
& -\int_{\mathbb{R}^{N}} H_{\lambda}(x, v) d x-\frac{1}{2^{*}} \int_{\mathbb{R}^{N}}|v|^{2^{*}} d x .
\end{aligned}
$$

Lemma 7. The functions $h_{\lambda}(x, s)$ and $H_{\lambda}(x, s)$ enjoy the following properties under $\left(f_{1}\right)-\left(f_{5}\right)$ :

(1) $\lim _{|s| \rightarrow 0}\left(h_{\lambda}(x, s) / s\right)=0$ and $\lim _{|s| \rightarrow 0}\left(H_{\lambda}(x, s) / s^{2}\right)=0$ uniformly in $x \in \mathbb{R}^{N}$;

(2) $\lim _{|s| \rightarrow \infty}\left(h_{\lambda}(x, s) /|s|^{2^{*}-1}\right)=0$ and $\lim _{|s| \rightarrow \infty}\left(H_{\lambda}(x, s) /\right.$ $\left.|s|^{2^{*}}\right)=0$ uniformly in $x \in \mathbb{R}^{N}$;
(3) $t h_{\lambda}(x, t)-2 H_{\lambda}(x, t) \geq s t h_{\lambda}(x, s t)-2 H_{\lambda}(x, s t)$ for all $t \in \mathbb{R}$ and $s \in[0,1]$

(4) $H_{\lambda}(x, s) \geq 0$ for all $(x, s) \in \mathbb{R}^{N} \times \mathbb{R}$;

(5) $\lim _{|s| \rightarrow+\infty}\left(H_{\lambda}(x, s) / s^{2}\right)=+\infty$ uniformly in $x \in \mathbb{R}^{N}$.

Proof. By $\left(f_{1}\right)-\left(f_{2}\right)$, for any $\varepsilon>0$, there exists $C_{\varepsilon}>0$ such that

$$
\left|\frac{f\left(x, G^{-1}(s)\right)}{g\left(G^{-1}(s)\right)}\right| \leq \varepsilon|s|+C_{\varepsilon}|s|^{p-1}
$$

for all $(x, s) \in \mathbb{R}^{N} \times \mathbb{R}$. Set $G^{-1}(s)=t$. Then Lemma 6(8) implies that

$$
\begin{aligned}
\lim _{|s| \rightarrow 0} \frac{h_{\lambda}(x, s)}{s} & =V(x)\left[1-\frac{1}{g^{2}(0)}\right]+\lambda \lim _{|t| \rightarrow 0} \frac{f(x, t)}{g(t) G(t)} \\
& =0
\end{aligned}
$$

uniformly in $x \in \mathbb{R}^{N}$. Moreover, by Lemma 6(6) one has

$$
\begin{aligned}
\lim _{|s| \rightarrow \infty} \frac{h_{\lambda}(x, s)}{|s|^{2^{*}-1}}= & -V(x) \lim _{|s| \rightarrow \infty} \frac{G^{-1}(s)}{s g\left(G^{-1}(s)\right)} \frac{s}{|s|^{2^{*}-1}} \\
& +\lambda \lim _{|t| \rightarrow \infty} \frac{f(x, t)}{g(t)|G(t)|^{2^{*}-1}}=0
\end{aligned}
$$

uniformly in $x \in \mathbb{R}^{N}$. Similarly, we have

$$
\lim _{|s| \rightarrow 0} \frac{H_{\lambda}(x, s)}{s^{2}}=0
$$

uniformly in $x \in \mathbb{R}^{N}$ and

$$
\lim _{|s| \rightarrow \infty} \frac{H_{\lambda}(x, s)}{|s|^{2^{*}}}=0
$$

uniformly in $x \in \mathbb{R}^{N}$. Hence, (1) and (2) hold.

In the following, we set $l(t)=G^{-1}(t)^{2}-G^{-1}(t) t / g\left(G^{-1}(t)\right)$, $\forall t \in \mathbb{R}$. If $t \geq 0$, by Lemma 6(2) and $g^{\prime}(t) \geq 0$ for $t \geq 0$, we have

$$
G(t)\left[\frac{1}{g^{2}(t)}\left(g(t)-g^{\prime}(t) t\right)\right] \leq t
$$

for $t \geq 0$, which implies that

$$
G(t)\left(\frac{t}{g(t)}\right)^{\prime} \frac{1}{g(t)} \leq \frac{t}{g(t)}
$$

for all $t \geq 0$. Let $r=G(t)$. Then

$$
G(t) \frac{d}{d r}\left(\frac{t}{g(t)}\right) \leq \frac{t}{g(t)}
$$

and hence

$$
r\left[\frac{G^{-1}(r)}{g\left(G^{-1}(r)\right)}\right]^{\prime} \leq \frac{G^{-1}(r)}{g\left(G^{-1}(r)\right)}
$$


for $r \geq 0$. Consequently,

$$
\begin{aligned}
l^{\prime}(t) & =\frac{2 G^{-1}(t)}{g\left(G^{-1}(t)\right)}-\left[\frac{G^{-1}(t)}{g\left(G^{-1}(t)\right)}\right]^{\prime} t-\frac{G^{-1}(t)}{g\left(G^{-1}(t)\right)} \\
& =\frac{G^{-1}(t)}{g\left(G^{-1}(t)\right)}-\left[\frac{G^{-1}(t)}{g\left(G^{-1}(t)\right)}\right]^{\prime} t \geq 0
\end{aligned}
$$

for all $t \geq 0$, that is, $l(t)$ is increasing with respect to $t \geq 0$. Hence $l(s t) \leq l(t)$ for all $s \in[0,1]$ and $t \geq 0$; that is,

$$
G^{-1}(s t)^{2}-\frac{G^{-1}(s t) s t}{g\left(G^{-1}(s t)\right)} \leq G^{-1}(t)^{2}-\frac{G^{-1}(t) t}{g\left(G^{-1}(t)\right)}
$$

for all $s \in[0,1]$ and $t \geq 0$. Note that Lemma 6(1) implies that $l(t)$ is an even function. Therefore, if $t<0$, we easily obtain that $l(s t) \leq l(t)$ for all $s \in[0,1]$ and $t<0$. Consequently,

$$
G^{-1}(s t)^{2}-\frac{G^{-1}(s t) s t}{g\left(G^{-1}(s t)\right)} \leq G^{-1}(t)^{2}-\frac{G^{-1}(t) t}{g\left(G^{-1}(t)\right)}
$$

for all $s \in[0,1]$ and $t \in \mathbb{R}$. Combining with $\left(f_{3}\right)$, we can conclude (3). Moreover, $\left(f_{4}\right)$ and Lemma 6(5) imply that $H(x, s) \geq 0$ for all $(x, s) \in \mathbb{R}^{N} \times \mathbb{R}$. Clearly, $\left(f_{5}\right)$ and Lemma 6(5) imply that (5) is satisfied. This completes the proof.

Lemma 8. Suppose that $\left(V_{1}\right),\left(V_{2}\right)$, and $\left(f_{1}\right)-\left(f_{2}\right)$ are satisfied. Then the energy functional $J_{\lambda}$ satisfies the following conditions:

(i) There exist $\beta, \rho>0$ such that $J_{\lambda}(v) \geq \beta$ for $\|v\|_{E}=\rho$.

(ii) There exists $e \in E$ with $\|e\|_{E}>\rho$ such that $J_{\lambda}(e)<0$.

Proof. (i) Set $S_{\rho}:=\left\{u \in E:\|u\|_{E}=\rho\right\}$. By $\left(f_{1}\right)-\left(f_{2}\right)$, Lemmas 6(6) and 7(1), and (2), for any $\varepsilon>0$, there exists $C_{\varepsilon}>0$ such that

$$
\left|H_{\lambda}(x, s)\right| \leq \varepsilon\left(|s|^{2}+|s|^{2^{*}}\right)+C_{\varepsilon}|s|^{p}
$$

for all $(x, s) \in \mathbb{R}^{N} \times \mathbb{R}$. Consequently, for $v \in S_{\rho}$, we have

$$
\begin{aligned}
J_{\lambda}(v) \geq & \frac{1}{2} C_{1}\|v\|_{E}^{2}-C_{2} \varepsilon\|v\|_{E}^{2}-C_{3} \varepsilon\|v\|_{E}^{2^{*}} \\
& -C_{4} C_{\varepsilon}\|v\|_{E}^{p} \\
\geq & \frac{1}{2} C_{1} \rho^{2}-C_{2} \varepsilon \rho^{2}-C_{3} \varepsilon \rho^{2^{*}}-C_{4} C_{\varepsilon} \rho^{p}:=\beta>0
\end{aligned}
$$

for small $\varepsilon>0$ and $\rho>0$.

(ii) Take $v^{*} \in E \backslash\{0\}$. Then

$$
\begin{aligned}
J_{\lambda}\left(t v^{*}\right) \leq & \frac{1}{2} C_{5} t^{2}\left\|v^{*}\right\|_{E}^{2}-\frac{1}{2^{*}} t^{2^{*}} \int_{\mathbb{R}^{N}}\left|v^{*}\right|^{2^{*}} d x \\
& +\varepsilon t^{2} \int_{\mathbb{R}^{N}}\left|v^{*}\right|^{2} d x+\varepsilon t^{2^{*}} \int_{\mathbb{R}^{N}}\left|v^{*}\right|^{2^{*}} d x \\
& +C_{\varepsilon} t^{p} \int_{\mathbb{R}^{N}}\left|v^{*}\right|^{p} d x<0
\end{aligned}
$$

for large $t>0$ and small $\varepsilon>0$. Consequently, we can take $e:=$ $t^{*} v^{*}$ for some large $t^{*}>0$ such that (ii) holds. This completes the proof.
Lemma 9. Suppose that $\left(V_{1}\right),\left(V_{2}\right)$, and $\left(f_{1}\right)-\left(f_{4}\right)$ are satisfied. Then there exists a bounded Cerami sequence $\left\{v_{n}\right\} \subset E$ for $J_{\lambda}$ with $J_{\lambda}\left(v_{n}\right) \rightarrow c_{\lambda} \geq \beta>0$, where

$$
\begin{aligned}
c_{\lambda}:=\inf _{\gamma \in \Gamma} \sup _{t \in[0,1]} J_{\lambda}(\gamma(t)), \\
\Gamma:=\left\{\gamma \in C([0,1], E): \gamma(0)=0, J_{\lambda}(\gamma(1))<0\right\},
\end{aligned}
$$

$\beta$ is the constant appearing in Lemma 8.

Proof. By Lemma 8 and the mountain pass theorem without (PS) condition (see Theorem 4.1 in [26]), there exists a Cerami sequence $\left\{v_{n}\right\} \subset E$ satisfying

$$
\begin{aligned}
J_{\lambda}\left(v_{n}\right) & \longrightarrow c_{\lambda} \geq \beta>0, \\
\left(1+\left\|v_{n}\right\|_{E}\right)\left\|J_{\lambda}^{\prime}\left(v_{n}\right)\right\|_{E^{*}} & \longrightarrow 0
\end{aligned}
$$

where

$$
\begin{aligned}
c_{\lambda}:= & \inf _{\gamma \in \Gamma} \sup _{t \in[0,1]} J_{\lambda}(\gamma(t)), \\
\Gamma & :=\left\{\gamma \in C([0,1], E): \gamma(0)=0, J_{\lambda}(\gamma(1))<0\right\},
\end{aligned}
$$

$\beta$ is the constant appearing in Lemma 8 .

Let $t_{n} \in[0,1]$ be such that $J_{\lambda}\left(t_{n} v_{n}\right)=\max _{t \in[0,1]} J_{\lambda}\left(t v_{n}\right)$. Then $\left\{J_{\lambda}\left(t_{n} v_{n}\right)\right\}$ is bounded from above. Indeed, without loss of the generality, we may assume that $t_{n} \in(0,1)$ for all $n \in \mathbb{N}$. Hence, by Lemma 7(3) we have

$$
\begin{aligned}
J_{\lambda}\left(t_{n} v_{n}\right)=J_{\lambda}\left(t_{n} v_{n}\right)-\frac{1}{2}\left\langle J_{\lambda}^{\prime}\left(t_{n} v_{n}\right), t_{n} v_{n}\right\rangle \\
=\left(\frac{1}{2}-\frac{1}{2^{*}}\right) t_{n}^{2^{*}} \int_{\mathbb{R}^{N}}\left|v_{n}\right|^{2^{*}} d x \\
\quad+\int_{\mathbb{R}^{N}}\left[\frac{1}{2} t_{n} v_{n} h_{\lambda}\left(x, t_{n} v_{n}\right)-H_{\lambda}\left(x, t_{n} v_{n}\right)\right] d x \\
\leq\left(\frac{1}{2}-\frac{1}{2^{*}}\right) \int_{\mathbb{R}^{N}}\left|v_{n}\right|^{2^{*}} d x \\
\quad+\int_{\mathbb{R}^{N}}\left[\frac{1}{2} v_{n} h_{\lambda}\left(x, v_{n}\right)-H_{\lambda}\left(x, v_{n}\right)\right] d x \\
=J_{\lambda}\left(v_{n}\right)-\frac{1}{2}\left\langle J_{\lambda}^{\prime}\left(v_{n}\right), v_{n}\right\rangle=c_{\lambda}+o(1) .
\end{aligned}
$$

This shows that $\left\{J_{\lambda}\left(t_{n} v_{n}\right)\right\}$ is bounded from above.

Now, we prove that $\left\{v_{n}\right\}$ is bounded in E. Otherwise, if $\left\|v_{n}\right\|_{E}$ is unbounded, then, up to a subsequence, we may assume that $\left\|v_{n}\right\|_{E} \rightarrow+\infty$. Set $w_{n}=v_{n} /\left\|v_{n}\right\|_{E}$. Then there exists $w \in E$ such that $w_{n} \rightarrow w$ in $E$. By $J_{\lambda}\left(v_{n}\right) \rightarrow c_{\lambda}$, we have

$$
\begin{aligned}
& o(1)+\frac{1}{2} \max \left\{1, V_{\infty}\right\} \\
& \geq \frac{1}{2^{*}} \frac{\left\|v_{n}\right\|_{2^{*}}^{2^{*}}}{\left\|v_{n}\right\|_{E}^{2}}+\int_{\mathbb{R}^{N}} \frac{H_{\lambda}\left(x, v_{n}\right)}{\left\|v_{n}\right\|_{E}^{2}} d x .
\end{aligned}
$$


Set $\Omega=\left\{x \in \mathbb{R}^{N}: w(x) \neq 0\right\}$. If meas $(\Omega)>0$, then by Lemma 7(4) and Fatou Lemma, one has

$$
\begin{aligned}
& o(1)+\frac{1}{2} \max \left\{1, V_{\infty}\right\} \\
& \geq \frac{1}{2^{*}} \frac{\left\|v_{n}\right\|_{2^{*}}^{2^{*}}}{\left\|v_{n}\right\|_{E}^{2}}+\int_{\mathbb{R}^{N}} \frac{H_{\lambda}\left(x, v_{n}\right)}{\left\|v_{n}\right\|_{E}^{2}} d x \\
& \geq \frac{1}{2^{*}} \int_{\Omega} w_{n}^{2}\left|v_{n}\right|^{2^{*}-2} d x \longrightarrow+\infty
\end{aligned}
$$

as $n \rightarrow \infty$. This is a contradiction. Hence $|\Omega|=0$, that is, $w=0$ a.e. on $\mathbb{R}^{N}$. For any $B>0$, by $\left\|v_{n}\right\|_{E} \rightarrow+\infty$ we have

$$
\begin{aligned}
J_{\lambda}\left(t_{n} v_{n}\right) \geq & J_{\lambda}\left(\frac{B}{\left\|v_{n}\right\|_{E}} v_{n}\right)=J_{\lambda}\left(B w_{n}\right) \\
\geq & \frac{B^{2}}{2} \min \left\{1, V_{0}\right\}-\int_{\mathbb{R}^{N}} H_{\lambda}\left(x, B w_{n}\right) d x \\
& -\frac{B^{2^{*}}}{2^{*}} \int_{\mathbb{R}^{N}}\left|w_{n}\right|^{2^{*}} d x
\end{aligned}
$$

for $n$ sufficiently large. By (29), Lemmas 6(6) and 7(1), and (2), for any $\varepsilon>0$, there exists $C_{\varepsilon}>0$ such that

$$
\left|h_{\lambda}(x, s) s\right| \leq \varepsilon\left(|s|^{2}+|s|^{2^{*}}\right)+C_{\varepsilon}|s|^{p}
$$

for all $(x, s) \in \mathbb{R}^{N} \times \mathbb{R}$. Consequently,

$$
\begin{aligned}
\int_{\mathbb{R}^{N}}\left|w_{n}\right|^{2^{*}} d x \leq & \frac{\max \left\{1, V_{\infty}\right\}}{\left\|v_{n}\right\|_{E}^{2^{*}-2}} \\
& -\frac{1}{\left\|v_{n}\right\|_{E}^{2^{*}}} \int_{\mathbb{R}^{N}} h_{\lambda}\left(x, v_{n}\right) v_{n} d x+o(1) \\
& \longrightarrow 0
\end{aligned}
$$

as $n \rightarrow \infty$ and so $\int_{\mathbb{R}^{N}}\left|w_{n}\right|^{p} d x \rightarrow 0$ as $n \rightarrow \infty$ by using interpolation inequality. Moreover, (41) implies that

$$
\begin{aligned}
\left|\int_{\mathbb{R}^{N}} H_{\lambda}\left(x, B w_{n}\right) d x\right| \leq & \varepsilon B^{2} \int_{\mathbb{R}^{N}} w_{n}^{2} d x \\
& +\varepsilon B^{2^{*}} \int_{\mathbb{R}^{N}}\left|w_{n}\right|^{2^{*}} d x \\
& +C_{\varepsilon} B^{p} \int_{\mathbb{R}^{N}}\left|w_{n}\right|^{p} d x
\end{aligned}
$$

By the arbitrariness of $\varepsilon$, we obtain $\int_{\mathbb{R}^{N}} H_{\lambda}\left(x, B w_{n}\right) d x \rightarrow 0$ as $n \rightarrow \infty$. Hence

$$
\liminf _{n \rightarrow \infty} J_{\lambda}\left(t_{n} v_{n}\right) \geq \frac{B^{2}}{2} \min \left\{1, V_{0}\right\}, \quad \forall B>0 .
$$

This contradicts the fact that $\left\{J_{\lambda}\left(t_{n} v_{n}\right)\right\}$ is bounded from above. Consequently, $\left\{v_{n}\right\}$ is bounded in $E$. This completes the proof of Lemma 9.
Lemma 10. Suppose that $\left(V_{1}\right),\left(V_{2}\right)$, and $\left(f_{1}\right)-\left(f_{5}\right)$ are satisfied. Then if $N \geq 5$, the minimax level $c_{\lambda}$ satisfies $c_{\lambda}<$ $(1 / N) S^{N / 2}$ for all $\lambda>0$; if $N=3,4$, the minimax level $c_{\lambda}$ satisfies $c_{\lambda}<(1 / N) S^{N / 2}$ for large $\lambda$, where $S$ is the best constant of the embedding $D^{1,2}\left(\mathbb{R}^{N}\right) \hookrightarrow L^{2^{*}}\left(\mathbb{R}^{N}\right)$.

Proof. From the minimax characterization of $c_{\lambda}$ we see that it is sufficient to show that there exists $v_{0} \in E \backslash\{0\}$ such that $\sup _{t \geq 0} J_{\lambda}\left(t v_{0}\right)<(1 / N) S^{N / 2}$.

We follow the strategy used in [24] but need to modify some process. Given $\varepsilon>0$, we consider the function

$$
w_{\varepsilon}(x)=\frac{[N(N-2) \varepsilon]^{(N-2) / 4}}{\left(\varepsilon+|x|^{2}\right)^{(N-2) / 2}}
$$

which satisfies the following equations:

$$
\begin{gathered}
-\Delta u=u^{2^{*}-1}, \quad \text { in } \mathbb{R}^{N}, \\
u \in D^{1,2}\left(\mathbb{R}^{N}\right), \\
u(x)>0,
\end{gathered}
$$

$$
\text { in } \mathbb{R}^{N} \text {. }
$$

Moreover, $w_{\varepsilon}(x)$ satisfies

$$
\left|\nabla w_{\varepsilon}\right|_{2}^{2}=\left|w_{\varepsilon}\right|_{2^{*}}^{2^{*}}=S^{N / 2}
$$

Let $\varphi \in C_{0}^{\infty}\left(\mathbb{R}^{N},[0,1]\right)$ be such that $\varphi(x) \equiv 1$ for $|x| \leq \rho_{\varepsilon}$ and $\varphi(x) \equiv 0$ for $|x| \geq 2 \rho_{\varepsilon}$, where $\rho_{\varepsilon}:=\varepsilon^{\tau}$ with $\tau \in(1 / 4,1 / 2)$. Set $\psi_{\varepsilon}(x)=\varphi(x) w_{\varepsilon}(x)$. Then

$$
\begin{aligned}
& \int_{\mathbb{R}^{N}}\left|\nabla \psi_{\varepsilon}\right|^{2} d x=S^{N / 2}+O\left(\varepsilon^{(N-2) / 2}\right), \\
& \int_{\mathbb{R}^{N}}\left|\psi_{\varepsilon}\right|^{2^{*}} d x=S^{N / 2}+O\left(\varepsilon^{N / 2}\right), \\
& \int_{\mathbb{R}^{N}}\left|\psi_{\varepsilon}\right| d x \leq C \varepsilon^{(N-2) / 4}, \\
& \int_{\mathbb{R}^{N}}\left|\psi_{\varepsilon}\right|^{2^{*}-1} d x \leq C \varepsilon^{(N-2) / 4}, \\
& \int_{\mathbb{R}^{N}}\left|\nabla \psi_{\varepsilon}\right| d x \leq C \varepsilon^{(N-2) / 4}, \\
& \int_{\mathbb{R}^{N}}\left|\psi_{\varepsilon}\right|^{2} d x= \begin{cases}C \varepsilon+O\left(\varepsilon^{(N-2) / 2}\right), & \text { if } N \geq 5, \\
C \varepsilon|\ln \varepsilon|+O(\varepsilon), & \text { if } N=4, \\
O\left(\varepsilon^{1 / 2}\right), & \text { if } N=3 .\end{cases}
\end{aligned}
$$

Since $J_{\lambda}(0)=0$ and $\lim _{t \rightarrow \infty} J_{\lambda}\left(t \psi_{\varepsilon}\right)=-\infty$, there exists $t_{\varepsilon}>0$ such that $J_{\lambda}\left(t_{\varepsilon} \psi_{\varepsilon}\right)=\max _{t \geq 0} J_{\lambda}\left(t \psi_{\varepsilon}\right)$. We claim that there exist two positive constants $t_{1}, t_{2}$ independent of $\varepsilon$ such that

$$
t_{1} \leq t_{\varepsilon} \leq t_{2}
$$


for small $\varepsilon>0$. Indeed, by $\left\langle J_{\lambda}^{\prime}\left(t_{\varepsilon} \psi_{\varepsilon}\right), \psi_{\varepsilon}\right\rangle=0$ we have

$$
\begin{array}{r}
\frac{\int_{\mathbb{R}^{N}}\left[\left|\nabla \psi_{\varepsilon}\right|^{2}+V(x) \psi_{\varepsilon}^{2}\right] d x}{\left|\psi_{\varepsilon}\right|_{2^{*}}^{2^{*}}}-t_{\varepsilon}^{2^{*}-2} \\
-\frac{\int_{\mathbb{R}^{N}} h_{\lambda}\left(x, t_{\varepsilon} \psi_{\varepsilon}\right) t_{\varepsilon} \psi_{\varepsilon} d x}{t_{\varepsilon}^{2}\left|\psi_{\varepsilon}\right|_{2^{*}}^{2^{*}}}=0 .
\end{array}
$$

By (29), Lemmas 6(6) and 7(1), and (2), for any $\delta>0$, there exists $C_{\delta}>0$ such that

$$
\left|h_{\lambda}(x, s) s\right| \leq \delta|s|^{2^{*}}+C_{\delta}|s|^{2}
$$

for all $(x, s) \in \mathbb{R}^{N} \times \mathbb{R}$. Consequently,

$$
\begin{aligned}
& \left|\frac{\int_{\mathbb{R}^{N}} h_{\lambda}\left(x, t_{\varepsilon} \psi_{\varepsilon}\right) t_{\varepsilon} \psi_{\varepsilon} d x}{t_{\varepsilon}^{2}\left|\psi_{\varepsilon}\right|_{2^{*}}^{2^{*}}}\right| \\
& \leq \frac{\int_{\mathbb{R}^{N}}\left[\delta t_{\varepsilon}^{2^{*}} \psi_{\varepsilon}^{2^{*}}+C_{\delta} t_{\varepsilon}^{2} \psi_{\varepsilon}^{2}\right] d x}{t_{\varepsilon}^{2}\left|\psi_{\varepsilon}\right|_{2^{*}}^{2^{*}}} \\
& =\delta t_{\varepsilon}^{2^{*}-2}+C_{\delta} \frac{\left|\psi_{\varepsilon}\right|_{2}^{2}}{\left|\psi_{\varepsilon}\right|_{2^{*}}^{2^{*}}} \\
& =\delta t_{\varepsilon}^{2^{*}-2}+C_{\delta}\left[S^{N / 2}+O\left(\varepsilon^{N / 2}\right)\right]^{-1}\left|\psi_{\varepsilon}\right|_{2}^{2} \\
& \leq \delta t_{\varepsilon}^{2^{*}-2}+C S^{-N / 2}\left|\psi_{\varepsilon}\right|_{2}^{2} \\
& =\delta t_{\varepsilon}^{2^{*}-2} \\
& +C S^{-N / 2} \begin{cases}C \varepsilon+O\left(\varepsilon^{(N-2) / 2}\right), & \text { if } N \geq 5 \\
C \varepsilon|\ln \varepsilon|+O(\varepsilon), & \text { if } N=4 \\
O\left(\varepsilon^{1 / 2}\right), & \text { if } N=3\end{cases} \\
& =\delta t_{\varepsilon}^{2^{*}-2}+o(1)
\end{aligned}
$$

as $\varepsilon \rightarrow 0$. Note that

$$
\begin{aligned}
& \frac{\left\|\psi_{\varepsilon}\right\|_{E}^{2}}{\left|\psi_{\varepsilon}\right|_{2^{*}}^{2^{*}}}=\frac{\left|\nabla \psi_{\varepsilon}\right|_{2}^{2}+\left|\psi_{\varepsilon}\right|_{2}^{2}}{\left|\psi_{\varepsilon}\right|_{2^{*}}^{2^{*}}}=\frac{1}{S^{N / 2}+O\left(\varepsilon^{N / 2}\right)} \\
& \qquad \begin{cases}S^{N / 2}+O\left(\varepsilon^{(N-2) / 2}\right)+C \varepsilon+O\left(\varepsilon^{(N-2) / 2}\right), & \text { if } N \geq 5, \\
S^{N / 2}+O\left(\varepsilon^{(N-2) / 2}\right)+C \varepsilon|\ln \varepsilon|+O(\varepsilon), & \text { if } N=4, \\
S^{N / 2}+O\left(\varepsilon^{(N-2) / 2}\right)+O\left(\varepsilon^{1 / 2}\right), & \text { if } N=3\end{cases}
\end{aligned}
$$$$
\longrightarrow 1
$$

as $\varepsilon \rightarrow 0$. Hence by (60) one has

$$
0 \geq \min \left\{1, V_{0}\right\}(1+o(1))-t_{\varepsilon}^{2^{*}-2}-\delta t_{\varepsilon}^{2^{*}-2}+o(1)
$$

as $\varepsilon \rightarrow 0$, which implies that

$$
t_{\varepsilon} \geq\left[\frac{\min \left\{1, V_{0}\right\}}{2(1+\delta)}\right]^{1 /\left(2^{*}-2\right)}:=t_{1}>0
$$

for $\varepsilon>0$ small enough. On the other hand, (60) leads to

$$
\begin{aligned}
t_{\varepsilon}^{2^{*}-2} \leq & \max \left\{1, V_{\infty}\right\} \frac{\left\|\psi_{\varepsilon}\right\|_{E}^{2}}{\left|\psi_{\varepsilon}\right|_{2^{*}}^{2^{*}}} \\
& +\left|\frac{\int_{\mathbb{R}^{N}} h_{\lambda}\left(x, t_{\varepsilon} \psi_{\varepsilon}\right) t_{\varepsilon} \psi_{\varepsilon} d x}{t_{\varepsilon}^{2}\left|\psi_{\varepsilon}\right|_{2^{*}}^{2^{*}}}\right| \\
\leq & \max \left\{1, V_{\infty}\right\}(1+o(1))+\delta t_{\varepsilon}^{2^{*}-2}+o(1)
\end{aligned}
$$

as $\varepsilon \rightarrow 0$, which implies that

$$
t_{\varepsilon} \leq\left[\frac{2 \max \left\{1, V_{\infty}\right\}}{1-\delta}\right]^{1 /\left(2^{*}-2\right)}:=t_{2}<+\infty
$$

for $\delta>0$ and $\varepsilon>0$ small enough.

Since $Q(t):=t^{2} / 2-t^{2^{*}} / 2^{*}$ has only maximum at $t=1$, one has

$$
\begin{aligned}
J_{\lambda}\left(t_{\varepsilon} \psi_{\varepsilon}\right)= & \frac{1}{2} t_{\varepsilon}^{2} \int_{\mathbb{R}^{N}}\left|\nabla \psi_{\varepsilon}\right|^{2} d x+\frac{1}{2} t_{\varepsilon}^{2} \int_{\mathbb{R}^{N}} V(x) \psi_{\varepsilon}^{2} d x \\
& -\int_{\mathbb{R}^{N}} H_{\lambda}\left(x, t_{\varepsilon} \psi_{\varepsilon}\right) d x \\
& -\frac{1}{2^{*}} t_{\varepsilon}^{2^{*}} \int_{\mathbb{R}^{N}} \psi_{\varepsilon}^{2^{*}} d x \\
= & \left(\frac{t_{\varepsilon}^{2}}{2}-\frac{t_{\varepsilon}^{2^{*}}}{2^{*}}\right) S^{N / 2}+O\left(\varepsilon^{(N-2) / 2}\right) \\
& +\frac{1}{2} t_{\varepsilon}^{2} \int_{\mathbb{R}^{N}} V(x) \psi_{\varepsilon}^{2} d x \\
& -\int_{\mathbb{R}^{N}} H_{\lambda}\left(x, t_{\varepsilon} \psi_{\varepsilon}\right) d x \\
\leq & \frac{1}{N} S^{N / 2}+O(\varepsilon(N-2) / 2) \\
& +\frac{1}{2} t_{2}^{2} V_{\infty} \int_{\mathbb{R}^{N}} \psi_{\varepsilon}^{2} d x \\
& -\int_{\mathbb{R}^{N}} H_{\lambda}\left(x, t_{\varepsilon} \psi_{\varepsilon}\right) d x
\end{aligned}
$$

Notice that, for $x \in B_{\rho_{\varepsilon}}$, we have

$$
\begin{aligned}
t_{\varepsilon} \psi_{\varepsilon} & =t_{\varepsilon} w_{\varepsilon}=t_{\varepsilon} \frac{[N(N-2) \varepsilon]^{(N-2) / 4}}{\left(\varepsilon+|x|^{2}\right)^{(N-2) / 2}} \\
& \geq C t_{\varepsilon} \frac{[N(N-2)]^{(N-2) / 4} \varepsilon^{(N-2) / 4}}{\varepsilon^{\tau(N-2)}} \\
& \geq C t_{1}[N(N-2)]^{(N-2) / 4} \varepsilon^{(N-2)(1 / 4-\tau)} \longrightarrow+\infty
\end{aligned}
$$

as $\varepsilon \rightarrow 0$, which combining with Lemma 7(4) and (5) implies that for any $M>0$

$$
\int_{\mathbb{R}^{N}} H_{\lambda}\left(x, t_{\varepsilon} \psi_{\varepsilon}\right) d x \geq M t_{\varepsilon}^{2} \int_{B_{\rho_{\varepsilon}}} \psi_{\varepsilon}^{2} d x
$$


for $\varepsilon>0$ small enough. Note that

$$
\begin{aligned}
& \int_{B_{\rho_{\varepsilon}}} \psi_{\varepsilon}^{2} d x \\
& \quad=[N(N-2)]^{(N-2) / 2} N \omega_{N} \varepsilon \int_{0}^{\rho_{\varepsilon} / \sqrt{\varepsilon}} \frac{s^{N-1}}{\left(1+s^{2}\right)^{N-2}} d s, \\
& \int_{0}^{+\infty} \frac{s^{N-1}}{\left(1+s^{2}\right)^{N-2}} d s \geq \int_{0}^{1} \frac{s^{N-1}}{\left(1+s^{2}\right)^{N-2}} d s \geq \frac{1}{N} \cdot \frac{1}{2^{N-2}} \\
& \quad:=C>0 .
\end{aligned}
$$

Consequently,

$$
\int_{\mathbb{R}^{N}} H_{\lambda}\left(x, t_{\varepsilon} \psi_{\varepsilon}\right) d x \geq M C \varepsilon
$$

for $\varepsilon>0$ small enough. Hence by (68)

$$
\begin{aligned}
J_{\lambda}\left(t_{\varepsilon} \psi_{\varepsilon}\right) \leq & \frac{1}{N} S^{N / 2}+O\left(\varepsilon^{(N-2) / 2}\right) \\
& +\frac{1}{2} t_{2}^{2} V_{\infty} \int_{\mathbb{R}^{N}} \psi_{\varepsilon}^{2} d x \\
& -\int_{\mathbb{R}^{N}} H_{\lambda}\left(x, t_{\varepsilon} \psi_{\varepsilon}\right) d x \\
\leq & \frac{1}{N} S^{N / 2}+O\left(\varepsilon^{(N-2) / 2}\right)-M C \varepsilon \\
& +C \begin{cases}\varepsilon+O\left(\varepsilon^{(N-2) / 2}\right), & \text { if } N \geq 5, \\
\varepsilon|\ln \varepsilon|+O(\varepsilon), & \text { if } N=4, \\
O\left(\varepsilon^{1 / 2}\right), & \text { if } N=3 .\end{cases}
\end{aligned}
$$

From this, we see that $J_{\lambda}\left(t_{\varepsilon} \psi_{\varepsilon}\right)<(1 / N) S^{N / 2}$ for $\varepsilon>0$ small enough and $M$ big enough if $N \geq 5$. Consequently, $c_{\lambda}<$ (1/ $N) S^{N / 2}$ for all $\lambda>0$ if $N \geq 5$.

In the following, we consider the case $N=3,4$. Indeed, if the conclusion is false, then there exists a sequence $\left\{\lambda_{n}\right\}$ with $\lambda_{n} \rightarrow+\infty$ such that $c_{\lambda_{n}} \geq(1 / N) S^{N / 2}$. Take $v \in E \backslash\{0\}$. Then by the proof of Lemma 8 , there exists a unique $t_{\lambda_{n}}>0$ such that $\max _{t>0} J_{\lambda_{n}}(t v)=J_{\lambda_{n}}\left(t_{\lambda_{n}} v\right)$. Hence

$$
\begin{aligned}
& t_{\lambda_{n}}^{2} \int_{\mathbb{R}^{N}}|\nabla v|^{2} d x+\int_{\mathbb{R}^{N}} V(x) \frac{G^{-1}\left(t_{\lambda_{n}} v\right)}{g\left(G^{-1}\left(t_{\lambda_{n}} v\right)\right)} t_{\lambda_{n}} v d x \\
& =t_{\lambda_{n}}^{2^{*}} \int_{\mathbb{R}^{N}}|v|^{2^{*}} d x \\
& \quad+\lambda_{n} \int_{\mathbb{R}^{N}} \frac{f\left(x, G^{-1}\left(t_{\lambda_{n}} v\right)\right)}{g\left(G^{-1}\left(t_{\lambda_{n}} v\right)\right)} t_{\lambda_{n}} v d x .
\end{aligned}
$$

By Lemma 6(6) and $\left(f_{4}\right)$ we get

$$
\max \left\{1, V_{\infty}\right\}\|v\|_{E}^{2} \geq t_{\lambda_{n}}^{2^{*}-2} \int_{\mathbb{R}^{N}}|v|^{2^{*}} d x,
$$

which implies that $\left\{t_{\lambda_{n}}\right\}$ is bounded. Hence, up to a subsequence, there exists $t_{0} \geq 0$ such that $t_{\lambda_{n}} \rightarrow t_{0}$ as $n \rightarrow \infty$. If $t_{0}>0$, then by $\left(f_{4}\right)$ and Fatou lemma we have

$$
\begin{aligned}
& \lim _{n \rightarrow \infty}\left[t_{\lambda_{n}}^{2^{*}} \int_{\mathbb{R}^{N}}|v|^{2^{*}} d x\right. \\
& \left.+\lambda_{n} \int_{\mathbb{R}^{N}} \frac{f\left(x, G^{-1}\left(t_{\lambda_{n}} v\right)\right)}{g\left(G^{-1}\left(t_{\lambda_{n}} v\right)\right)} t_{\lambda_{n}} v d x\right]=+\infty .
\end{aligned}
$$

But, on the other hand, by Lemma 6(6) one has

$$
\begin{aligned}
& t_{\lambda_{n}}^{2^{*}} \int_{\mathbb{R}^{N}}|v|^{2^{*}} d x+\lambda_{n} \int_{\mathbb{R}^{N}} \frac{f\left(x, G^{-1}\left(t_{\lambda_{n}} v\right)\right)}{g\left(G^{-1}\left(t_{\lambda_{n}} v\right)\right)} t_{\lambda_{n}} v d x \\
& \quad \leq \max \left\{1, V_{\infty}\right\} t_{\lambda_{n}}^{2}\|v\|_{E}^{2} \longrightarrow \max \left\{1, V_{\infty}\right\} t_{0}^{2}\|v\|_{E}^{2},
\end{aligned}
$$

a contradiction. Hence $t_{0}=0$ and by Lemma 7(4) we know that

$$
\begin{aligned}
\max _{t>0} J_{\lambda_{n}}(t v)= & J_{\lambda_{n}}\left(t_{\lambda_{n}} v\right) \\
\leq & \frac{1}{2} \max \left\{1, V_{\infty}\right\} t_{\lambda_{n}}^{2}\|v\|_{E}^{2} \\
& -\frac{1}{2^{*}} t_{\lambda_{n}}^{2^{*}} \int_{\mathbb{R}^{N}}|v|^{2^{*}} d x \longrightarrow 0
\end{aligned}
$$

as $n \rightarrow \infty$. Consequently,

$$
\begin{aligned}
0 & <\frac{1}{N} S^{N / 2} \leq c_{\lambda_{n}} \leq \inf _{u \in E \backslash\{0\}} \max _{t>0} J_{\lambda_{n}}(t u) \leq \max _{t>0} J_{\lambda_{n}}(t v) \\
& \longrightarrow 0
\end{aligned}
$$

a contradiction. This completes the proof.

Proof of Theorem 1. Since $\left\{v_{n}\right\} \subset E$ is a bounded Cerami sequence for $J_{\lambda}$ at the level $c_{\lambda}>0$, there exists $v \in E$ such that

$$
\begin{gathered}
v_{n} \rightarrow v \quad \text { in } E, \\
v_{n} \longrightarrow v \quad \text { in } L_{\text {loc }}^{q}\left(\mathbb{R}^{N}\right) \text { for } 1 \leq q<2^{*}, \\
v_{n}(x) \longrightarrow v(x) \quad \text { a.e. on } \mathbb{R}^{N} .
\end{gathered}
$$

Using a standard argument, we know that $J_{\lambda}^{\prime}(v)=0$, that is, $v$ is a weak solution of (11). Indeed, for any $\psi \in C_{0}^{\infty}\left(\mathbb{R}^{N}\right)$, we have

$$
\begin{aligned}
o(1)= & \left\langle J_{\lambda}^{\prime}\left(v_{n}\right), \psi\right\rangle \\
= & \int_{\mathbb{R}^{N}} \nabla v_{n} \nabla \psi d x+\int_{\mathbb{R}^{N}} V(x) v_{n} \psi d x \\
& -\int_{\mathbb{R}^{N}}\left|v_{n}\right|^{2^{*}-2} v_{n} \psi d x-\int_{\mathbb{R}^{N}} h_{\lambda}\left(x, v_{n}\right) \psi d x .
\end{aligned}
$$


Advances in Mathematical Physics

9

Since $v_{n} \rightarrow v$ in $E$, one has

$$
\begin{gathered}
\int_{\mathbb{R}^{N}} \nabla v_{n} \nabla \psi d x \rightarrow \int_{\mathbb{R}^{N}} \nabla v \nabla \psi d x, \\
\int_{\mathbb{R}^{N}} V(x) v_{n} \psi d x \longrightarrow \int_{\mathbb{R}^{N}} V(x) v \psi d x, \\
\int_{\mathbb{R}^{N}}\left|v_{n}\right|^{2^{*}-2} v_{n} \psi d x \longrightarrow \int_{\mathbb{R}^{N}}|v|^{2^{*}-2} v \psi d x, \\
\int_{\mathbb{R}^{N}} h_{\lambda}\left(x, v_{n}\right) \psi d x \rightarrow \int_{\mathbb{R}^{N}} h_{\lambda}(x, v) \psi d x .
\end{gathered}
$$

Consequently,

$$
\begin{aligned}
0= & \int_{\mathbb{R}^{N}} \nabla v \nabla \psi d x+\int_{\mathbb{R}^{N}} V(x) v \psi d x \\
& -\int_{\mathbb{R}^{N}}|v|^{2^{*}-2} v \psi d x-\int_{\mathbb{R}^{N}} h_{\lambda}(x, v) \psi d x
\end{aligned}
$$

for all $\psi \in C_{0}^{\infty}\left(\mathbb{R}^{N}\right)$. For any $\varphi \in E$, there exists a sequence $\left\{\psi_{n}\right\} \subset C_{0}^{\infty}\left(\mathbb{R}^{N}\right)$ such that $\psi_{n} \rightarrow \varphi$ in $E$. Hence

$$
\begin{aligned}
0= & \int_{\mathbb{R}^{N}} \nabla v \nabla \psi_{n} d x+\int_{\mathbb{R}^{N}} V(x) v \psi_{n} d x \\
& -\int_{\mathbb{R}^{N}}|v|^{2^{*}-2} v \psi_{n} d x-\int_{\mathbb{R}^{N}} h_{\lambda}(x, v) \psi_{n} d x .
\end{aligned}
$$

Let $n \rightarrow \infty$, we get

$$
\begin{aligned}
0= & \int_{\mathbb{R}^{N}} \nabla v \nabla \varphi d x+\int_{\mathbb{R}^{N}} V(x) v \varphi d x \\
& -\int_{\mathbb{R}^{N}}|v|^{2^{*}-2} v \varphi d x-\int_{\mathbb{R}^{N}} h_{\lambda}(x, v) \varphi d x ;
\end{aligned}
$$

that is, $\left\langle J_{\lambda}^{\prime}(v), \varphi\right\rangle=0$ for all $\varphi \in E$. Hence $J_{\lambda}^{\prime}(v)=0$; that is, $v$ is a weak solution of (11).

In the following, we prove that $v$ is nontrivial. With the aid of Lemma 10, the proof follows essentially the proof of Theorem 1.1 in [16]. For completeness, we present the proof as follows. If the conclusion is false, we may assume $v=0$. We divide the proof into four steps.

Step 1. We prove that $\left\{v_{n}\right\} \subset E$ is also a Ceram sequence for the functional $J_{\lambda}^{\infty}: E \rightarrow \mathbb{R}$, where

$$
\begin{aligned}
J_{\lambda}^{\infty}\left(v_{n}\right)= & \frac{1}{2} \int_{\mathbb{R}^{N}}\left[\left|\nabla v_{n}\right|^{2}+V_{\infty} v_{n}^{2}\right] d x \\
& -\int_{\mathbb{R}^{N}} H_{\lambda}\left(x, v_{n}\right) d x-\frac{1}{2^{*}} \int_{\mathbb{R}^{N}}\left|v_{n}\right|^{2^{*}} d x .
\end{aligned}
$$

By $\left(V_{2}\right)$ and $v_{n} \rightarrow 0$ in $E$, one has

$$
J_{\lambda}\left(v_{n}\right)-J_{\lambda}^{\infty}\left(v_{n}\right)=\frac{1}{2} \int_{\mathbb{R}^{N}}\left[V(x)-V_{\infty}\right] v_{n}^{2} d x \longrightarrow 0
$$

as $n \rightarrow \infty$. Similarly, we have

$$
\begin{aligned}
& \left\|J_{\lambda}^{\prime}\left(v_{n}\right)-\left(J_{\lambda}^{\infty}\right)^{\prime}\left(v_{n}\right)\right\|_{E^{*}} \\
& =\sup _{\|\varphi\|_{E} \leq 1}\left|\left\langle J_{\lambda}^{\prime}\left(v_{n}\right)-\left(J_{\lambda}^{\infty}\right)^{\prime}\left(v_{n}\right), \varphi\right\rangle\right| \\
& =\sup _{\|\varphi\|_{E} \leq 1}\left|\int_{\mathbb{R}^{N}}\left[V(x)-V_{\infty}\right] v_{n} \varphi d x\right| \longrightarrow 0
\end{aligned}
$$

as $n \rightarrow \infty$. Consequently, $\left\{v_{n}\right\}$ is also a Ceram sequence of $J_{\lambda}^{\infty}$.

Step 2. There exist $\alpha, R>0$ and $\left\{y_{n}\right\} \subset \mathbb{R}^{N}$ such that

$$
\lim _{n \rightarrow \infty} \int_{B_{R}\left(y_{n}\right)}\left|v_{n}\right|^{2} d x \geq \alpha>0 .
$$

Indeed, by contradiction, then by Lemma 1.21 in [27], one has $v_{n} \rightarrow 0$ in $L^{q}\left(\mathbb{R}^{N}\right)$ for $2<q<2^{*}$. Notice that

$$
\begin{aligned}
o(1)= & \left\langle J_{\lambda}^{\prime}\left(v_{n}\right), v_{n}\right\rangle \\
= & \int_{\mathbb{R}^{N}}\left[\left|\nabla v_{n}\right|^{2}+V(x) v_{n}^{2}\right] d x \\
& -\int_{\mathbb{R}^{N}} h_{\lambda}\left(x, v_{n}\right) v_{n} d x-\int_{\mathbb{R}^{N}}\left|v_{n}\right|^{2^{*}} d x,
\end{aligned}
$$

which combining with (51) leads to

$$
\int_{\mathbb{R}^{N}}\left[\left|\nabla v_{n}\right|^{2}+V(x) v_{n}^{2}\right] d x-\int_{\mathbb{R}^{N}}\left|v_{n}\right|^{2^{*}} d x \longrightarrow 0
$$

as $n \rightarrow \infty$. Consequently, there exists a constant $l \geq 0$ such that

$$
\begin{array}{r}
\int_{\mathbb{R}^{N}}\left[\left|\nabla v_{n}\right|^{2}+V(x) v_{n}^{2}\right] d x \rightarrow l, \\
\int_{\mathbb{R}^{N}}\left|v_{n}\right|^{2^{*}} d x \longrightarrow l .
\end{array}
$$

Obviously, $l>0$. Otherwise, $J_{\lambda}\left(v_{n}\right) \rightarrow 0$ as $n \rightarrow \infty$, which contradicts with $c_{\lambda}>0$. Hence by the definition of $S$, we have

$$
\begin{aligned}
S & \leq \frac{\int_{\mathbb{R}^{N}}\left|\nabla v_{n}\right|^{2} d x}{\left(\int_{\mathbb{R}^{N}}\left|v_{n}\right|^{2^{*}} d x\right)^{2 / 2^{*}}} \\
& \leq \frac{\int_{\mathbb{R}^{N}}\left[\left|\nabla v_{n}\right|^{2}+V(x) v_{n}^{2}\right] d x}{\left(\int_{\mathbb{R}^{N}}\left|v_{n}\right|^{2^{*}} d x\right)^{2 / 2^{*}}} \rightarrow \frac{l}{l^{2 / 2^{*}}}=l^{2 / N} ;
\end{aligned}
$$

that is, $l \geq S^{N / 2}$. Therefore, (41) implies that

$$
\begin{aligned}
c_{\lambda}+o(1)= & J_{\lambda}\left(v_{n}\right) \\
= & \frac{1}{2} \int_{\mathbb{R}^{N}}\left[\left|\nabla v_{n}\right|^{2}+V(x) v_{n}^{2}\right] d x \\
& -\int_{\mathbb{R}^{N}} H_{\lambda}\left(x, v_{n}\right) d x-\frac{1}{2^{*}} \int_{\mathbb{R}^{N}}\left|v_{n}\right|^{2^{*}} d x \\
& \longrightarrow\left(\frac{1}{2}-\frac{1}{2^{*}}\right) l=\frac{1}{N} l \geq \frac{1}{N} S^{N / 2},
\end{aligned}
$$


as $n \rightarrow \infty$, which implies that $c_{\lambda} \geq(1 / N) S^{N / 2}$, a contradiction.

Step 3. After a translation of $\left\{v_{n}\right\}$ called $\left\{\widetilde{v}_{n}\right\}$, then $\widetilde{v}_{n}$ converges weakly to a nonzero critical point of $J_{\lambda}^{\infty}$.

Set $\widetilde{v}_{n}(x)=v_{n}\left(x+y_{n}\right)$. Since $\left\{v_{n}\right\} \subset E$ is a Cerami sequence of $J_{\lambda}^{\infty}$ and $\left\|\widetilde{v}_{n}\right\|_{E}=\left\|v_{n}\right\|_{E}$, arguing as in the case of $\left\{v_{n}\right\}$, we may assume $\widetilde{v}_{n} \rightarrow \widetilde{v}$ in $E$ and $\left(J_{\lambda}^{\infty}\right)^{\prime}(\widetilde{v})=0$. So by Step 2 we know $\widetilde{v} \neq 0$. By Lemma 7(3) and Fatou Lemma, one has

$$
\begin{aligned}
2 c_{\lambda}= & \liminf _{n \rightarrow \infty}\left[2 J_{\lambda}^{\infty}\left(\widetilde{v}_{n}\right)-\left\langle\left(J_{\lambda}^{\infty}\right)^{\prime}\left(\widetilde{v}_{n}\right), \widetilde{v}_{n}\right\rangle\right] \\
\geq & \liminf _{n \rightarrow \infty} \int_{\mathbb{R}^{N}}\left[h_{\lambda}\left(x, \widetilde{v}_{n}\right) \widetilde{v}_{n}-2 H_{\lambda}\left(x, \widetilde{v}_{n}\right)\right] d x \\
& +\left(1-\frac{2}{2^{*}}\right) \liminf _{n \rightarrow \infty} \int_{\mathbb{R}^{N}}\left|\widetilde{v}_{n}\right|^{2^{*}} d x \\
\geq & \int_{\mathbb{R}^{N}}\left[h_{\lambda}(x, \widetilde{v}) \widetilde{v}-2 H_{\lambda}(x, \widetilde{v})\right] d x \\
& +\left(1-\frac{2}{2^{*}}\right) \int_{\mathbb{R}^{N}}|\widetilde{v}|^{2^{*}} d x \\
= & 2 J_{\lambda}^{\infty}(\widetilde{v})-\left\langle\left(J_{\lambda}^{\infty}\right)^{\prime}(\widetilde{v}), \widetilde{v}\right\rangle=2 J_{\lambda}^{\infty}(\widetilde{v})
\end{aligned}
$$

which implies that $J_{\lambda}^{\infty}(\widetilde{v}) \leq c_{\lambda}$.

Step 4. We use $\widetilde{v}$ to construct a path which allows us to obtain a contradiction with the definition of mountain pass level $c_{\lambda}$.

Define the mountain pass level $c_{\lambda}^{\infty}:=$ $\inf _{\gamma \in \Gamma_{\infty}} \sup _{t \in[0,1]} J_{\lambda}^{\infty}(\gamma(t))>0$, where $\Gamma_{\infty}:=\{\gamma \in C([0,1], E)$ : $\left.\gamma(0)=0, J_{\lambda}^{\infty}(\gamma(1))<0\right\}$. It follows the arguments used in $[28,29]$, we can construct a path $\gamma:[0,1] \rightarrow E$ such that

$$
\begin{aligned}
\gamma(0) & =0, \\
J_{\lambda}^{\infty}(\gamma(1)) & <0, \\
\widetilde{v} & \in \gamma([0,1]), \\
\gamma(t)(x) & >0, \quad \forall x \in \mathbb{R}^{N}, t \in[0,1], \\
\max _{t \in[0,1]} J_{\lambda}^{\infty}(\gamma(t)) & =J_{\lambda}^{\infty}(\widetilde{v}) .
\end{aligned}
$$

Then $c_{\lambda}^{\infty} \leq \max _{t \in[0,1]} J_{\lambda}^{\infty}(\gamma(t))=J_{\lambda}^{\infty}(\widetilde{v})$. If $V(x) \equiv V_{\infty}$, we have already proved Theorem 1. If $V(x) \leq V_{\infty}$ but $V(x) \quad \equiv$ $V_{\infty}$, we take the path $\gamma$ given by above, and by $\gamma \in \Gamma_{\infty} \subset \Gamma$, we have

$$
\begin{aligned}
c_{\lambda} & \leq \max _{t \in[0,1]} J_{\lambda}(\gamma(t))=J_{\lambda}(\gamma(\bar{t}))<J_{\lambda}^{\infty}(\gamma(\bar{t})) \\
& \leq \max _{t \in[0,1]} J_{\lambda}^{\infty}(\gamma(t))=J_{\lambda}^{\infty}(\widetilde{v}) \leq c_{\lambda},
\end{aligned}
$$

a contradiction. Consequently, $v \neq \equiv$. This completes the proof of Theorem 1 .

\section{Conflicts of Interest}

The authors declare that there are no conflicts of interest regarding the publication of this paper.

\section{Acknowledgments}

This work is supported in part by the National Natural Science Foundation of China (11501403; 11461023; 11701322), the Shanxi Province Science Foundation for Youths under Grant 2013021001-3, the Honghe University Doctoral Research Programs (XJ17B11 and XJ17B12), the Yunnan Province Applied Basic Research for Youths, and the Yunnan Province Local University (Part) Basic Research Joint Project.

\section{References}

[1] S. Kurihara, "Large-Amplitude Quasi-Solitons in Superfluid Films," Journal of the Physical Society of Japan, vol. 50, no. 10, pp. 3262-3267, 1981.

[2] E. W. Laedke, K. H. Spatschek, and L. Stenflo, "Evolution theorem for a class of perturbed envelope soliton solutions," Journal of Mathematical Physics, vol. 24, no. 12, pp. 2764-2769, 1983.

[3] H. S. Brandi, C. Manus, G. Mainfray, T. Lehner, and G. Bonnaud, "Relativistic and ponderomotive self-focusing of a laser beam in a radially inhomogeneous plasma. I. Paraxial approximation," Physics of Fluids, vol. 5, no. 10, pp. 3539-3550, 1993.

[4] X. L. Chen and R. N. Sudan, "Necessary and sufficient conditions for self-focusing of short ultraintense laser pulse in underdense plasma," Physical Review Letters, vol. 70, no. 14, pp. 2082-2085, 1993.

[5] A. de Bouard, N. Hayashi, and J.-C. Saut, "Global existence of small solutions to a relativistic nonlinear Schrödinger equation," Communications in Mathematical Physics, vol. 189, no. 1, pp. 73105, 1997.

[6] B. Ritchie, "Relativistic self-focusing and channel formation in laser-plasma interactions," Physical Review E: Statistical, Nonlinear, and Soft Matter Physics, vol. 50, no. 2, pp. R687-R689, 1994.

[7] L. Brüll and H. Lange, "Solitary waves for quasilinear Schrödinger equations," Expositiones Mathematicae. International Journal for Pure and Applied Mathematics, vol. 4, no. 3, pp. 279-288, 1986.

[8] F. G. Bass and N. N. Nasonov, "Nonlinear electromagnetic-spin waves," Physics Reports, vol. 189, no. 4, pp. 165-223, 1990.

[9] V. G. Makhankov and V. K. Fedyanin, "Non-linear effects in quasi-one-dimensional models of condensed matter theory," Physics Reports, vol. 104, no. 1, pp. 1-86, 1984.

[10] R. W. Hasse, "A general method for the solution of nonlinear soliton and kink Schrödinger equations," Zeitschrift für Physik. B. Condensed Matter and Quanta, vol. 37, no. 1, pp. 83-87, 1980.

[11] H. Lange, M. Poppenberg, and H. Teismann, "Nash-Moser methods for the solution of quasilinear Schrödinger equations," Communications in Partial Differential Equations, vol. 24, no. 78, pp. 1399-1418, 1999.

[12] A. M. Kosevich, B. A. Ivanov, and A. S. Kovalev, "Magnetic Solitons," Physics Reports, vol. 194, no. 3-4, pp. 117-238, 1990.

[13] M. Poppenberg, K. Schmitt, and Z.-Q. Wang, "On the existence of soliton solutions to quasilinear Schrödinger equations," Calculus of Variations and Partial Differential Equations, vol. 14, no. 3, pp. 329-344, 2002.

[14] G. R. Quispel and H. W. Capel, "Equation of motion for the Heisenberg spin chain," Physica. A. Europhysics Journal, vol. 110, no. 1-2, pp. 41-80, 1982. 
[15] S. Cuccagna, "On instability of excited states of the nonlinear Schrödinger equation," Physica D: Nonlinear Phenomena, vol. 238, no. 1, pp. 38-54, 2009.

[16] Y. Shen and Y. Wang, "Soliton solutions for generalized quasilinear Schrödinger equations," Nonlinear Analysis. Theory, Methods \& Applications. An International Multidisciplinary Journal, vol. 80, pp. 194-201, 2013.

[17] W. H. Fleming, "A selection-migration model in population genetics," Journal of Mathematical Biology, vol. 2, no. 3, pp. 219233, 1975.

[18] P. L. Kelley, "Self-focusing of optical beams," Physical Review Letters, vol. 15, no. 26, pp. 1005-1008, 1965.

[19] Q. Li, K. Tang, and X. Wu, "Ground state solutions and geometrically distinct solutions for generalized quasilinear Schrödinger equation," Mathematical Methods in the Applied Sciences, vol. 40, no. 6, pp. 2165-2176, 2017.

[20] Q. Li and X. Wu, "Multiple solutions for generalized quasilinear Schrödinger equations," Mathematical Methods in the Applied Sciences, vol. 40, no. 5, pp. 1359-1366, 2017.

[21] Q. Li and X. Wu, "Existence, multiplicity, and concentration of solutions for generalized quasilinear Schrödinger equations with critical growth," Journal of Mathematical Physics, vol. 58, no. 4, 041501, 30 pages, 2017.

[22] Q. Li and X. Wu, "Existence of nontrivial solutions for generalized quasilinear Schrödinger equations with critical or supercritical growths," Acta Mathematica Scientia B, vol. 37, no. 6, pp. 1870-1880, 2017.

[23] M. Colin and L. Jeanjean, "Solutions for a quasilinear Schrödinger equation: a dual approach," Nonlinear Analysis. Theory, Methods \& Applications. An International Multidisciplinary Journal, vol. 56, no. 2, pp. 213-226, 2004.

[24] Y. Deng, S. Peng, and S. Yan, "Positive soliton solutions for generalized quasilinear Schrödinger equations with critical growth," Journal of Differential Equations, vol. 258, no. 1, pp. 115$147,2015$.

[25] Y. Ye and C.-L. Tang, "Infinitely many solutions for fourthorder elliptic equations," Journal of Mathematical Analysis and Applications, vol. 394, no. 2, pp. 841-854, 2012.

[26] J. F. Aires and M. A. Souto, "Existence of solutions for a quasilinear Schrödinger equation with vanishing potentials," Journal of Mathematical Analysis and Applications, vol. 416, no. 2, pp. 924-946, 2014.

[27] M. Willem, Minimax Theorems, Birkhäuser, Boston, Mass, USA, 1996.

[28] J. M. Bezerra do Ó, O. m. Miyagaki, and S. H. Soares, "Soliton solutions for quasilinear Schrödinger equations with critical growth," Journal of Differential Equations, vol. 248, no. 4, pp. 722-744, 2010.

[29] L. Jeanjean and K. Tanaka, "A remark on least energy solutions in $\boldsymbol{R}^{N}$," Proceedings of the American Mathematical Society, vol. 131, no. 8, pp. 2399-2408, 2003. 


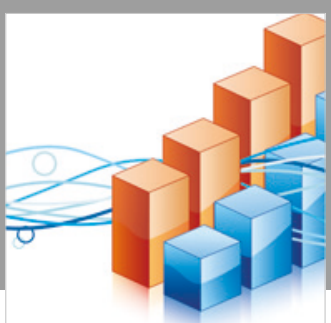

Advances in

Operations Research

\section{-n-m}
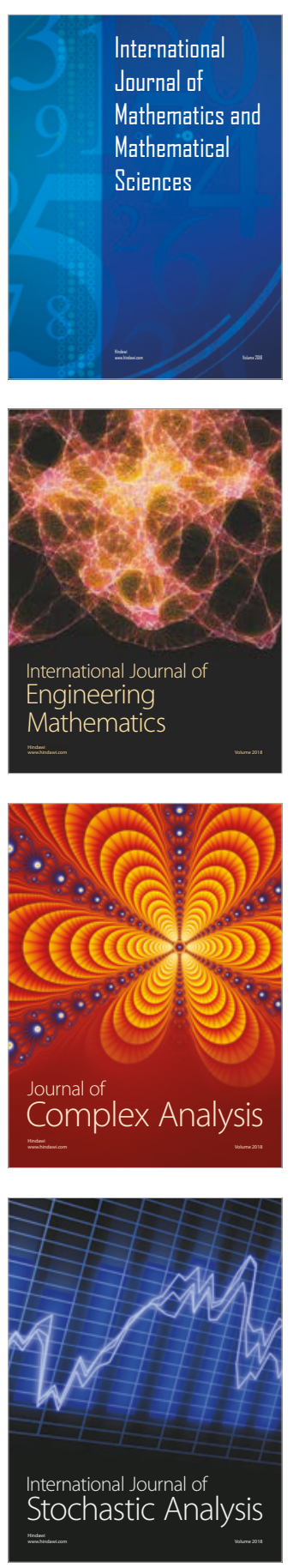
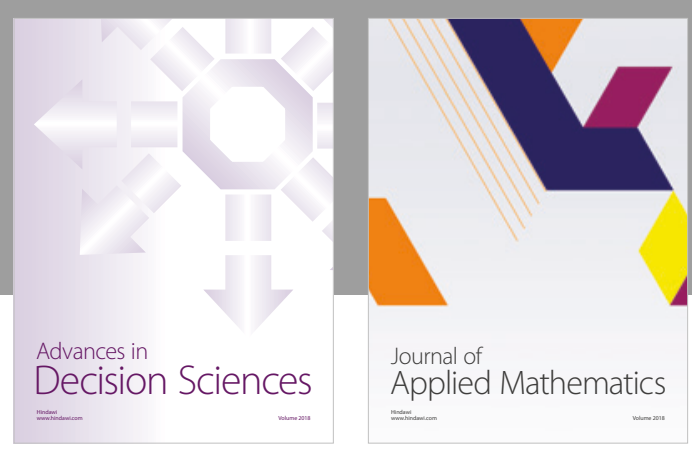

Journal of

Applied Mathematics
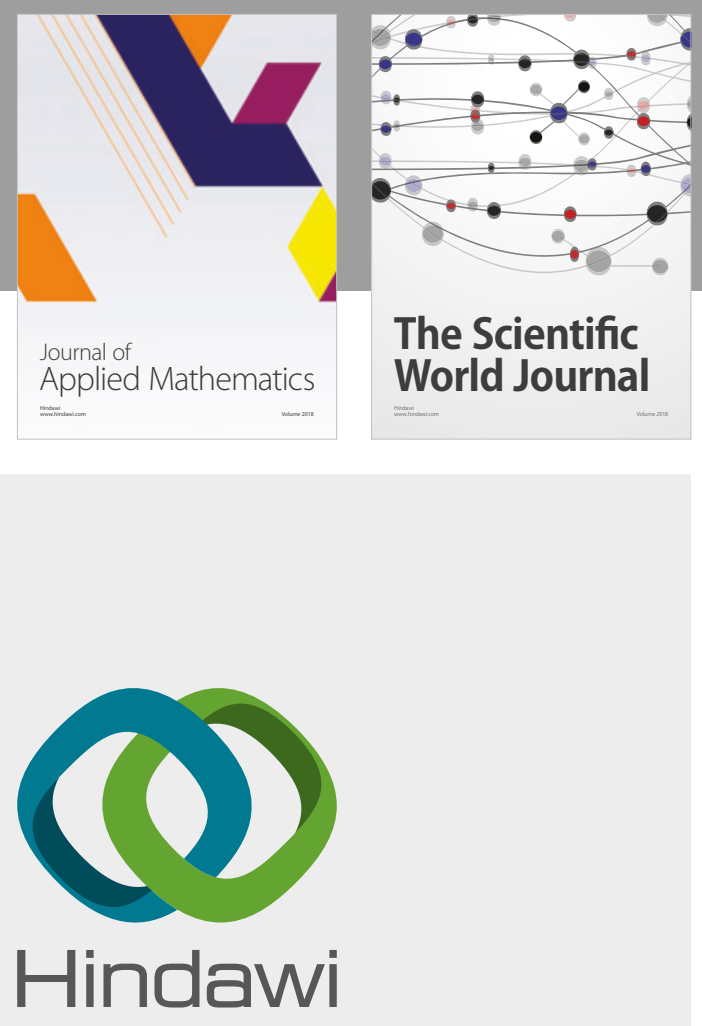

Submit your manuscripts at

www.hindawi.com

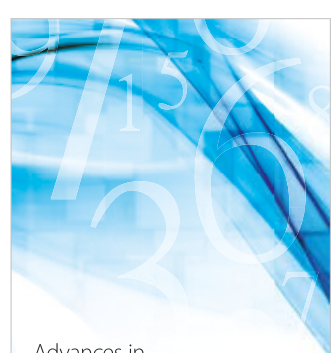

Advances in
Numerical Analysis
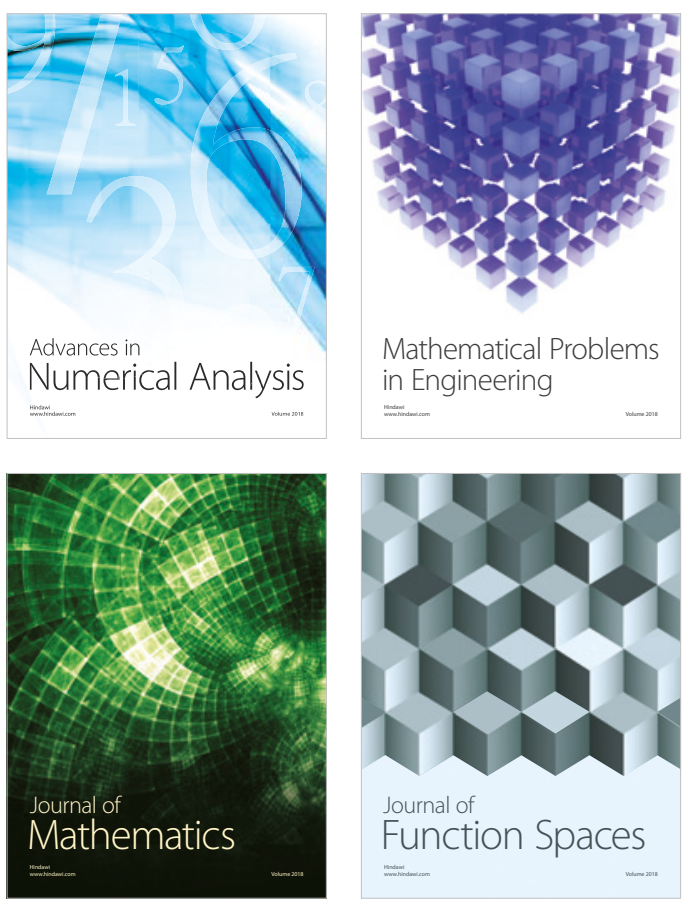

Mathematical Problems in Engineering

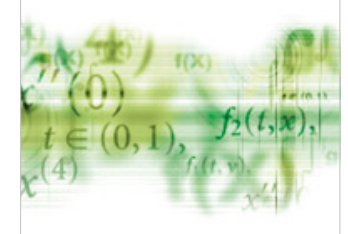

International Journal of

Differential Equations

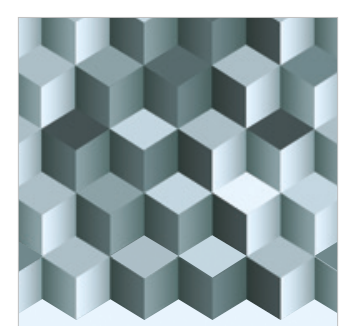

Journal of

Function Spaces

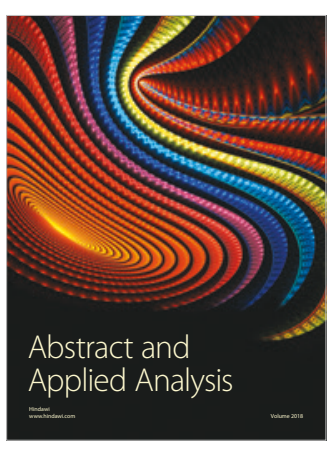

The Scientific

World Journal

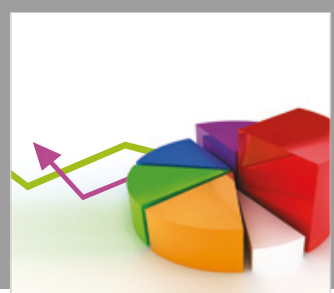

Journal of

Probability and Statistics
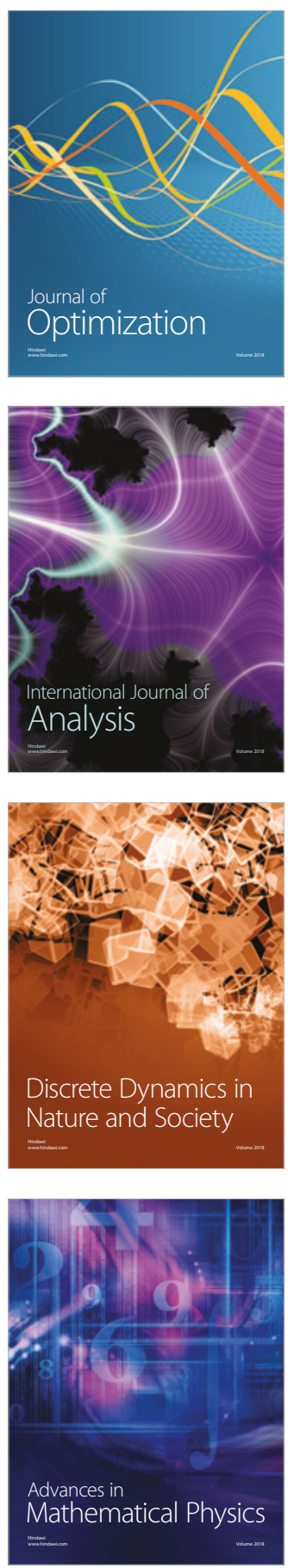